\title{
NONABELIAN COUNTEREXAMPLES TO THE NOETHER PROBLEM
}

\author{
JACK SONN
}

\begin{abstract}
Let $G$ be a finite group having a cyclic quotient of order 8 . Let $L$ be the field given by adjoining indeterminates $\left\{x_{g} \mid g \in G\right\}$ to $\mathbf{Q}$. Then $L^{G}$ is not a rational function field.
\end{abstract}

Let $G$ be a finite group, $\left\{x_{g}: g \in G\right\}$ a set of commuting indeterminates and $L$ the rational function field over $\mathbf{Q}$ in the $x_{g}$. Then $G$ acts as a group of automorphisms of $L$ by the rule $x_{g}^{h}=x_{g h}, g, h \in G$. The "Noether problem" is whether or not $L^{G}$ is a rational function field. This question was first given a negative answer by Swan [3] for certain cyclic groups $G$, the smallest being of order 47 . For abelian groups the question was solved completely by Lenstra [1] and recently Saltman [2] has shed a new light on the question via his theory of generic Galois extensions. Lenstra classified all abelian $G$ for which $L^{G}$ is rational and in particular obtained a smallest negative example: $L^{G}$ is not rational for $G$ a cyclic group of order 8 . Saltman obtains the nonrationality of $L^{G}$ for this group as well as for any abelian group having an element of order 8 [2, Theorem 5.11]. There do not appear to be examples of nonrational $L^{G}$ for nonabelian $G$ in the literature. The purpose of this note is to make the observation that Saltman's results generate nonabelian examples as well as abelian ones determined earlier by Lenstra.

We begin by pointing out that the proof of Saltman's theorem [2, Theorem 5.11] on the nonrationality of $L^{G}$ for abelian groups having an element of order 8 actually applies to any split extension

$$
1 \rightarrow N \rightarrow G \rightarrow C \rightarrow 1,
$$

where $C$ is cyclic of 2 -power order $\geq 8$.

We therefore have

THEOREM 1 (SALTMAN). Let $G$ be a split extension (1) with $C$ cyclic of 2 power order $\geq 8$. Then there is no generic extension for $G$ over $\mathbf{Q}$ and therefore $L^{G}$ is nonrational.

The hypothesis in Theorem 1-that the extension (1) splits-can be dropped. Namely we have

THEOREM 2. Let $G$ be a finite group having a cyclic factor group of order 8 . Then $L^{G}$ is nonrational.

Proof. Let $N$ be a normal subgroup of $G$ with $G / N$ cyclic of order 8 . Then $G$ has a cyclic subgroup $C$ of 2 -power order $\geq 8$ such that $G=C N$. Suppose $L^{G}$ is rational. Then by [2, Theorem 5.1], $G$ has a generic extension over $\mathbf{Q}$;

Received by the editors January 3, 1984.

1980 Mathematics Subject Classification. Primary 12A55; Secondary 12F20, 13 B05.

(C) 1985 American Mathematical Society $0002-9939 / 85 \$ 1.00+\$ .25$ per page 
hence, by $[\mathbf{2}$, Theorem 5.9], $G$ has the "Grünwald property" over Q: let there be given subgroups $H_{1}, \ldots, H_{m}$ of $G$ having the property that any subgroup of $G$ containing one conjugate of each of the $H_{i}$ must be all of $G$. (Let us say that such a set of subgroups conjugate interchangeably generates $G$.) Suppose that $p_{1}, \ldots, p_{m}$ are distinct primes, $\mathbf{Q}_{p_{i}}$ the field of $p_{i}$-adic rationals and $L_{i} / \mathbf{Q}_{p_{i}}$ Galois (field) extensions with $G\left(L_{i} / \mathbf{Q}_{p_{i}}\right) \simeq H_{i}, i=1, \ldots, n$. Then there exists a Galois (field) extension $L / \mathbf{Q}$ such that the completion of $L$ at a divisor of $p_{i}$ in $L$ coincides with $L_{i}$, and $H_{i}$ is the corresponding decomposition group, $i=1, \ldots, n$. (We do not go into an explanation of the notion of generic extension since all we need is that $L^{G}$ rational implies that $G$ has the Grünwald property.)

Now let $C_{1}, \ldots, C_{n}$ be all the cyclic subgroups of $G$, and let $C_{1}=C$. Then $C_{1}, \ldots, C_{n}$ conjugate interchangeably generate $G$. Indeed, if not, then there would be a proper subgroup $G_{1}$ of $G$ having the property that every element of $G$ has a conjugate in $G_{1}$, or equivalently, every element of $G$ lies in a conjugate of $G_{1}$, which is impossible for $G_{1}$ a proper subgroup of $G$. Now let $p_{1}=2$ and let $p_{2}, \ldots, p_{n}$ be any distinct odd primes. Let $n_{i}=\left|C_{i}\right|, i=1, \ldots, n$. Let $L_{i} / \mathbf{Q}_{p_{i}}$ be the unramified extension of degree $n_{i}$. Then the Grünwald property implies that there exists a Galois extension $L / \mathbf{Q}$ with group $G$ such that $L_{p_{i}}=L_{i}$, where $L_{p_{i}}$ is the completion of $L$ at a divisor of $p_{i}$ in $L$, and $C_{i}$ is the corresponding decomposition group, $i=1, \ldots, n$.

Let $K$ be the fixed field of $N . K / \mathbf{Q}$ is cyclic of degree 8. 2 is unramified in $K$ because 2 is unramified in $L$. Let $F$ be the decomposition field of a prime $v$ of $L$ dividing 2, where we may assume that $F$ is the fixed field of $C$. Since $C N=G$, we have $F \cap K=\mathbf{Q}$. Hence $F K / F$ is cyclic of degree 8. Let $w$ be the prime of $F$ below $v$. Then $w$ remains prime in $L$, hence in $F K$, so $\left[(F K)_{u}: F_{w}\right]=8$, where $u$ is the prime of $F K$ below $v$ (and above $w$ ). But $F_{w}=\mathbf{Q}_{2}$, and $(F K)_{u}=F_{w} K_{y}=K_{y}$, where $y$ is the prime of $K$ below $v$. Hence $\left[K_{y}: \mathbf{Q}_{2}\right]=8$. Thus $K / \mathbf{Q}$ is cyclic of degree 8 and localizes at 2 to the unramified extension of degree 8 , which is impossible (Wang's counterexample to Grünwald's Theorem [4]). Q.E.D.

\section{REFERENCES}

1. H. W. Lenstra, Rational functions invariant under a finite abelian group, Invent. Math. 25 (1974), 299-325.

2. D. Saltman, Generic Galois extensions and problems in field theory, Adv. in Math. 43 (1982), 250--283.

3. R. Swan, Invariant rational functions and a problem of Steenrod, Invent. Math. 7 (1969), 148-158.

4. S. Wang, A counterexample to Grünwald's Theorem, Ann. of Math. (2) 49 (1948), 1008-1009.

Department of Mathematics, Technion-Israel Institute of Technology, HAIFA 32000 , ISRAEL 